\title{
Presentism as an empirical hypothesis
}

\begin{abstract}
Within philosophy of physics it is broadly accepted that presentism as an empirical hypothesis has been falsified by the development of special relativity. In this paper, I identify and reject an assumption common to both presentists and advocates of the block universe, and then offer an alternative version of presentism that does not begin from spatiotemporal structure, which is an empirical hypothesis, and which has yet to be falsified. I fear that labelling it "presentism” dooms the view, but I don’t know what else to call it.
\end{abstract}

\section{Introduction}

Here are two premises:

(P1) All and only things that exist now are real.

(P2) Special relativity is a complete account of spatiotemporal structure.

The first is a version of so-called "presentism”. It says that what is real is what exists right now: this is what there is. Things in the past aren't real (they don’t exist any more); things in the future aren’t real (they don't exist yet). What there is, is what is present. "Now" bears a lot of ontological weight. The second says that there are no good reasons for adding anything to the account of space and time found in 
Einstein's special theory of relativity: it's complete. The problem for people attracted to presentism is that (P2) seems to be incompatible with (P1), for reasons that I'll come to below. The dominant view amongst philosophers of physics is that we should therefore reject (P1) and adopt the so-called "block universe”, a four-dimensional structure where everything that has ever existed, and ever will exist, is all equally real. There is no "now” of any ontological significance, there’s just the whole four-dimensional shebang. That's what there is. What Einstein showed in developing special relativity is that, even if there is such a thing as “the present”, we have no empirical access to it. If there is any evidence for presentism, then it does not come from empirical experience. There is nothing in empirical experience that supports this concept; there is no empirically well-grounded concept of "the present". Presentism, if treated as the hypothesis that there is such a concept, is false.

For philosophers of physics such as myself, this "block universe” is the default position, the “well yeah, of course” point of view, according to which anyone who’s a presentist hasn’t learned the hard-won lessons from physics properly. End of story.

However, in this paper I argue for an alternative empirical approach to "the present”, equally well founded in physics as Einstein's treatment of simultaneity, according to which the dispute between presentists and block universe people remains an open empirical question, not decided by special relativity. In other words, even if you endorse (P2), there’s a form of presentism that remains a live option. I was sufficiently surprised to find myself reaching this conclusion that I decided I should write it down.

\section{Why presentism is false}

Let's go back to the two premises above, (P1) and (P2), and remind ourselves why they appear not to work very well together. According to Howard Stein (in a 1968 paper responding to Putnam’s 1967 
paper on special relativity), adopting both (P1) and (P2) leads to "the interesting result that special relativity implies a peculiarly extreme (but pluralistic) form of solipsism.” The reason for this is familiar from the literature on space and time. Given an event e1 in spacetime, e1 is "now" relative to itself, but there is nothing within the structure of special relativistic spacetime that determines which events spatiotemporally distant from e1 are also "now" relative to e1. There’s no preferred way to join the dots and say these two events are both "now”. You can conclude this directly from conventionality of simultaneity, in which case any “joining of the dots” in planes of simultaneity is an addition, going beyond the content of special relativity. Or you can get there via relativity of simultaneity: adopt the Einstein synchrony convention, note that different planes of simultaneity make different determinations of which events are "now" relative to e1 and which are not, note that picking one of these - a preferred plane of simultaneity - goes beyond the content of special relativity, and so conclude that no other events are determinately “now” with respect to e1. Either way, the conclusion is that there’s no preferred way to join the dots.

If we focus our attention on (P2), and ignore (P1), this argument typically leads to endorsing the block universe. What special relativity gives us is just the entire set of events, arranged in a fourdimensional block.

But if we want to have (P1) as well as (P2), we get a different conclusion. If no other events are determinately "now" with respect to e1, then, by (P1), no other events are determinately "real” with respect to e1. Add to that the claim that everything that is real must be determinately so, and we get our conclusion: nothing is real with respect to e1 except e1 itself (hence the extreme solipsism), although each event is real with respect to itself (hence the pluralism). This is Stein's pluralistic extreme solipsism.

"Pluralistic extreme solipsism” is what you get if you hang on to presentism as expressed in (P1), and to (P2). So far as I know, the view hasn’t attracted many adherents, and I suppose that's not very 
surprising. Instead, the standard moves in the interpretation of special relativity reject either (P1) or (P2). On the one hand, there are those who suggest that "taking our experience of time seriously" requires us to reject, or supplement, special relativity. So we accept (P1), and in an effort to avoid the slide towards extreme solipsism, we reject (P2), perhaps trying to stay as close as possible to special relativity but adding a preferred foliation so that we get a unique, global "now". On the other hand there are those who suggest that "taking special relativity seriously" requires us to give up presentism. We endorse (P2) and straightfowardly reject -- throw out -- (P1). We adopt the four-dimensional "block universe”. And we characterize our presentist opponents as intellectual cowards, clinging to their unfounded pre-critical intuitions in the face of overwhelming evidence from the conceptual developments wrought by science. There are lots of variations, lots of ways of trying to finesse things, but those are the basic moves.

I am in agreement with the proponents of the "block universe” in this dispute, as I have set it up. I don’t think there is some "every day” concept of time that we can make use of philosophically and that's independent of the scientific concept. Science starts from everyday experience and investigates those very concepts, clarifying and changing them along the way. DiSalle makes this point very beautifully in his book Understanding spacetime. The book is about the engagement of physics with our concepts of space and time: the way that developments in physics have brought about developments in those very concepts, and how there’s no “other” concept of time that's independent of these developments and somehow left standing untouched by them.

Presentism as expressed by (P1), and in appropriately similar versions, as an empirical hypothesis is false.

\section{A shared assumption}


Below, I argue for an alternative formulation of presentism, as an empirical hypothesis, such that the considerations of simultaneity just discussed in section 2 do not lead to the conclusion that presentism is false. I will not do this on the basis of our "experience of time”. Rather, I will make the argument from within physics itself. I endorse (P2): special relativity gives us a complete account of spatiotemporal structure. I will not add a preferred foliation or anything like that; I think that’s entirely misguided. Instead what we need to do is investigate the conceptual development that has taken place, with philosophy and physics taken together, working hand-in-hand, and when we do that we see that there is a presentist alternative available, and one which will not be “pluralistic extreme solipsism”.

The reason why I reject “pluralistic extreme solipsism” is not just distaste, but because there is an assumption in the interpretation of special relativity that's common to both the "block universe" and the presentist positions characterized above, which I think the presentist should reject. The reason why "pluralistic extreme solipsism” follows from adopting both (P1) and (P2) is that space-time is being used by both of the block universe people and the presentists as a principle of ontological unity. This is the "shared assumption” to be rejected, and it is a familiar claim according to which space and time provide the ontological framework within which everything that is material exists. Or, to put it another way, spacetime is the ground of the unity of the world: what makes this material universe one universe is the shared space and time framework within which the matter is located.

For those of us interested in modern science, this approach to the unity of the world has a venerable pedigree. In Newton’s physics, space and time can be understood as playing just such a role.

With this assumption explicitly on the table, let us revisit (P1). (P1) attempts to ground the unity of what exists, of what is real, in simultaneity. All and only things that exist now are real. If the "now" of a given real thing extends to other things, then those other things are also real. The unity of the real is grounded in their simultaneity. That's okay, if there's absolute simultaneity. But if "now" is not spatially extended, then what is real - given $\mathrm{P}(1)$ - is not spatially extended either. As discussed above, special 
relativity doesn't underwrite a spatial extension of “now” via absolute simultaneity and so, given a commitment to special relativity as asserted by (P2), we arrive at pluralistic extreme solipsism.

What's gone wrong is that we bought into the proposal that spacetime is the ontological ground of the unity of what there is. My argument begins from the observation that we don't have to use spacetime to play this role. In what follows, I reject the shared assumption and develop an alternative formulation of presentism accordingly.

\section{An alternative ontological principle of unity}

Something that I won't argue for in this paper, but will simply state, is that Newton's physics contains alternatives to space and time as the ontological ground of the unity of what there is. One of these grounds unity in the three laws of motion. According to this approach, we begin not with space and time, but with the laws, which are viewed as providing a principle of unity. The proposal is that we don't begin with the physical entities, as given unities, and then ask about the laws that they satisfy. Rather, the laws themselves play a constitutive role in constituting the very entities that are their subject-matter, and in constituting them as genuine unities. Thus, to be a physical thing, be it simple or composite, is (in part) to conserve total quantity of motion (when isolated from other physical things), this being required by Newton’s laws. Moreover, the laws also play a constitutive role with respect to the parts of a composite physical entity. Consider, for example, a collision between two billiard balls: not only is the

total quantity of motion conserved (so the composite is a genuine unity), but that quantity is redistributed determinately, and from the law-constitutive perpective this is what makes the billiard balls themselves genuine unities throughout the process. The unity of the whole, and the unity of each part, is grounded in the laws. I cannot argue for this view here, for that would be another paper in itself. I make use of the view in what follows, in discussing presentism, and in so doing will further elaborate it 
somewhat. One important aspect of the view will be its treatment of change. Changes in the state of a component are determined by the laws, so the laws provide an account of what it is for a genuine unity (the part) to undergo change whilst remaining the very same thing. Saying the same thing another way: the laws provide an account of what it is for a unity to persist through change: that is, to retain its numerical identity whilst not its qua litative identity. It does this without appeal to either essential properties or to haecceities. It offers us an alternative and one which, I am going to argue, favors a version presentism that is an empirical claim, but one which is distinct from that explored by Einstein in his 1905 paper on special relativity. For now, I am simply making a claim: there is in Newton’s physics an alternative option for a principle of unity, based on the laws, and this should be on the table as something that we discuss and evaluate.

\section{Change}

What is it for an object to persist through change? The prima facie puzzle here is as old as it is familiar. How can a thing - by which we mean a genuine unity - remain the very same thing and yet undergo change? In particular, if $\mathrm{F}$ and $\mathrm{G}$ are inconsistent properties (e.g. being 5 inches long and being 7 inches long), then (1) Fa, (2) Gb, and (3) a=b cannot all be true (something cannot be both 5 and 7 inches long). How might one respond?

On the one hand, one might hold fast to the principle that no genuine unity can have inconsistent properties, and conclude that no genuine unity in fact persists through change at all. No numerical identity without qualitative identity. Thus, we make the distinction between enduring unities and perduring unities, and insist that objects persist in virtue of perduring (through a succession of momentary genuine unities appropriately related to one another), not in virtue of enduring. 
On the other hand, we might take seriously the idea that time is doing some important work here, and allow that while a genuine unity cannot have inconsistent properties at any one time, having inconsistent properties at different times might be tolerated somehow (in a way that is to be explicitly specified). So, we allow for the possibility of numerical identity in the absence of qualitative identity. Since numercal unity cannot be grounded in qualitative identity on this route, we must ground it in something else, and there are two prominent options. One might restrict the class of properties that are required to remain the same in order for the numerical identity of the thing to be preserved: the essential properties do not change, no object has associated with it a set of inconsistent essential properties, not even over time. As for the accidental properties, we require that these are consistent at any one time, but we do not to care whether they contain inconsistencies over time. Alternatively, one might claim that numerical identity over time is independent of sameness of properties over time: we appeal to haecceities to ground numerical identity over time, and we don’t care about any inconsistencies in properties over time (although we continue to require that an object's properties be consistent at any one time). This allows for genuine unities which persist in virtue of enduring.

There are good reasons for philosophers of physics to be sceptical about both essentialism and haecceitism, which appears to leave "no numerical identity without qualitative identity" as a feature of our account of unity, and consequently perdurantism as our account of change, as the only option. But the law-constitutive approach reveals an alternative. The law-constitutive approach offers a principle of unity in virtue of which a thing remains the very same thing over time and through change of properties. It does so not by appeal to haecceities, nor by appeal to essential properties, but by specifying the relations that must hold between the states of the thing at different times. You might think: okay, but this view is compatible with both perdurantism and endurantism: for the perdurantist the laws specify the relationship between the successive momentary genuine unities for the endurantist the laws specify the relationship between successive states of a single genuine unity. But this isn’t right. Here’s the crucial 
question: what are the perdurantist’s “momentary genuine unities” that are supposedly tied together by the laws? In virtue of what are these - the things that are tied together - themselves genuine unities? If the genuine unity is grounded in qualitative identity, give me an argument why I should accept this view. If it's grounded in something else, tell me what. In the absence of an answer to this, I think that the law-constitutive approach gives us an argument in favor of endurantism as against perdurantism, because according to the law-constitutive person the very principle that grounds the unity of a thing has as one of its consequences rules by which such a unity can undergo qualitative change.

This is a key point, and for that reason I want to emphasize it a little further. In generating the prima facie puzzle about change, we had to write down “a=b”. But in order to write this down, we have to presuppose that our things labelled by “a” and "b” are genuine unities, and we need an account of what grounds that unity. We can’t take unity as brute, at least not without saying why the worries of the seventeenth century philosophers were misplaced. ${ }^{1}$ So, in the absence of a principle of unity, suitably argued for, the perdurantist is at a disadvantage as compared to the endurantist. The law-constitutive approach offers a principle of unity which provides numerical identity without qualitative identity, and it provides an account of what it is for a genuine unity to undergo change. It's an approach that arose within attempts to construct a physics and a metaphysics of things by two giants of this enterprise: Descartes and Newton. What it gives us is a reason to prefer endurantism, and from here it is perhaps a short step to presentism, for while both endurantism and perdurantism are compatible with both presentism and four-dimensionalism, most metaphysicians think there is a more natural fit between

\footnotetext{
${ }^{1}$ In our theorizing about the world, objects should not be taken as primitives. As Saunders (2003) argues, we have access (at least in physics) first of all not to objects, but to their properties and relations, and (for the purposes of physics at least) identity of objects needs to be defined in these terms, not taken as primitive. For example, Della Rocca (forthcoming) diagnoses an apparent stand-off between endurantism and perdurantism, and then argues against endurantism on the grounds that the endurantist must take persistence as primitive. The implicit assumption Della Rocca makes is that objects are to be taken as primitives: in the law-constitutive approach, objects are not primitive, and neither is persistence. Thus, endurantism escapes Della Rocca’s argument.
} 
endurantism and presentism and between perdurantism and four-dimensionalism. If that's right, then the law-constitutive approach to unity and change favors presentism.

This leaves us with a tension. On the one hand, considerations arising out of space-time theory push strongly towards the block universe. On the other hand, consideration arising out of Newton’s physics lead to a form of presentism. In the final sections of my paper I attempt to remove this tension, and in so doing I further elaborate the alternative form of presentism that I take to be both (a) an empirical hypothesis, and (b) compatible with special relativity.

\section{Space and time as an epistemic principle of unity}

At the end of section 3, I claimed that we don't have to use spacetime to play the role of an ontological principle of unity. In section 4 I offered a sketch of an alternative, and in section 5 I showed how this alternative favors a version of presentism. If we are to take this route (and I fully concede that it stands in great need of significant further elaboration), then we should re-visit the status of spacetime. If it no longer serves as a principle of ontological unity, what role does it play? Why do we set out a big arena of space and time when we're doing physics? The answer, I think, is that spacetime plays the role of an epistemic principle of unity, as follows. In mechanics, we want to know what the outcome of a collision will be, before it happens, based on knowledge of events prior to the collision. Quite generally, one thing we're doing is trying to extend our knowledge of events to times and places distant from the here and now. Space\&time play a theoretical role, as we try to extend our epistemic reach beyond the herenow, stitching our predictions together into a single whole. Thinking of things in this way, space\&time provide an epistemic principle of unity: they provide the framework in which we organize our knowledge of the not-here and/or not-now. 
There is no necessary inference from this epistemic role for spacetime to the view that spacetime is an ontological principle of unity. Thus, if we take this route, we should revise our understanding of (P2). In being committed to special relativity as a complete account of spatiotemporal structure, we do not thereby automatically ontologize this structure: we recognize its epistemic status and we do not make any direct inference from that to any ontological commitments.

Instead, we adopt the approach sketched in section 4, according to which the laws provide the principle of ontological unity. Thus, whatever spatiotemporal ontological commitments we have must come from paying attention to the details of the dynamical laws of matter. Matter is spatiotemporal, but it's not in space and time in the sense that space and time provide an ontological principle of unity for what there is. ${ }^{2}$

According to this approach, the dynamical laws ground the unity of a thing, and the spatiotemporal extent of that thing is whatever size it needs to be in order to sustain the dynamically characterized thing in question. Once this approach is adopted, (P2) means two things:

(1) We take special relativity seriously as an epistemic principle of unity: this is the best way of organizing our knowledge that reaches beyond the here-now.

(2) Ontologically, what there is is grounded in the dynamical laws, and these include the spatiotemporal characteristics of things. If things are spatiotemporally extended, then special relativity tells us that within that spacetime region there are no purely spatial or purely temporal relations. Things “occupy” time just as they “occupy” space: by existing as a unity that is spatiotemporally extended. Notice that the spatiotemporal extent of the sufficient dynamical ground of a given unity might turn out to be much, much smaller than the abstract spatiotemporal structure within which the evolution of that unity is

\footnotetext{
${ }^{2}$ Ontologically, space and time are not independent of bodies. But this is not relationism either, so we move immediately to a middle way between ab space and time versus relationism. (This is a second reason for the superiority of the dynamical approach.)
} 
completely described, or it might be the size of the block universe. ${ }^{3}$ This is an empirical matter, something to be settled by the progress of science. I will return to this point in a moment.

\section{An alternative "presentism"}

What becomes of presentism on this view? (P1) will need to be re-written. The reason is that, on the approach being developed here, we are not going to start from Minkowkski spacetime when we do ontology. Hence, the presentist should not use "now" as the grounds of what is real: she should use dynamics instead. The present is a spatiotemporal region of whatever size is necessary to sustain the dynamical system in question. ${ }^{4}$ If we take this route, then we will not be driven by our considerations of the structure of Minkowski spacetime to the conclusion that the present is merely a point in Minkowski spacetime, and that therefore this is all that is real. In other words, since we are not using a "now" derived from the structure of Minkowksi spacetime to ground what is real, we don't end up at pluralistic extreme solipsism.

It might turn out that, for whatever system we try to consider, the size of the spatiotemporal region necessary to sustain it is the entire history of the universe, encompassing all that ever has been and ever will be. In other words, the only dynamical system that there is, is this entirety, and there are no genuine subsystems of the universe. If that turns out to be the case, then this version of presentism is defeated and the block universe triumphs. But notice that this is an empirical matter, something to be decided by consideration of the details of physics, and perhaps science more generally. Quantum entanglement might give us good reason to think that this is true, but if these consideration lead to the

\footnotetext{
${ }^{3}$ I can be made of things whose dynamically sustained regions of spacetime and much smaller than mine, so they come into and go out of existence on much shorter timescales than I do. That seems fine to me. (There's always something existing whenever I exist - it's hard to say this right, I can see this is going to be an interesting challenge to express this thesis!)

${ }^{4}$ There are no determinate ontological spatial or temporal relations within that region, and that system stands in no determinate ontological spatial or temporal relations to any other system. All the ontology is carried by the dynamics, and we frame the dynamics spatiotemporally, but ontologically the dynamics requires no such spatiotemporal underpinning.
} 
defeat of presentism then they do so via a different route than Einstein's treatment of simultaneity. Notice also, however, that this issue is not yet settled.

So let's begin from the position that there are genuine subsystems. This means that the size of the spatiotemporal region required to sustain the system is less than the entire block universe, and so the present is (at least in the first instance) local, not global. This "local now" does not lead to solipsism, however, because it is not the ground of what is real. The grounds of what is real is the dynamics, and we belong to the same world as whatever we interact with, and the rest of that world is as real as we are. The dynamics grounds the unity of what there is, both of the parts and of the whole (consisiting of interacting parts), and this is what prevents the presentist from becoming a solipsist. Does my son exist

relative to me, when he is in London and I am here? Of course. There are plenty of interactions going on that link us. Is there a determinate fact of the matter about what he is doing right now? No.

Clearly, there is a lot of work to be done in filling out exactly what this position says. But one thing we can do straight away is reformulate presentism such that it doesn't ground the reality of what exists in spacetime. Here’s an attempt at a better (P1):

$\left(\mathrm{P} 1^{*}\right)$ For each and every thing, that thing exists only presently, where the spatiotemporal extent of that “present” is dependent on dynamics, and it is something to be determined empirically

This is a version of presentism that endorses (P2).

\section{Conclusion}

That we can systematize things in a global spatiotemporal framework is surely an interesting fact, and you might want to ontologize the overall framework above and beyond the dynamics. You can if you 
want to. My point has been that you don't have to, and that if you're going to you need to say why adopting spacetime as the ground of ontological unity is better than using the dynamical laws. Should you choose to adopt dynamical laws as the ground of unity, an alternative version of presentism emerges, and one which (unlike the version grounded in spacetime structure) has yet to be empirically falsified. A great deal rests on whether there are genuine subsystems of the universe, and that's something that we can find out only through empirical enquiry.

\section{References}

Della Rocca, Michael. Forthcoming.

DiSalle, Robert. 2006. Understanding Space-Time: The philosophical development of physics from Newton to Einstein. Cambridge: Cambridge University Press.

Saunders, Simon. 2003. Physics and Leibniz's Principles. Symmetries in Physics: Philosophical

Reflections, K. Brading and E. Castellani, eds., Cambridge University Press.

Stein, Howard. 1968. On Einstein-Minkowski Space-Time. Journal of Philosophy 65(1):5-23.

\section{4,380 words}

